\title{
Misunderstanding Data on Time: a Cautionary Tale
}

\author{
Maurizio Rossi*, Marco Trentini* \\ *Department of Education Studies, University of Bologna
}

\begin{abstract}
This article offers a different perspective to that usually used to interpret data on time use. According to current interpretations, the indicators used-mainly generic average duration, specific average duration, the frequency of participation - provide information on the behavior of the individuals in the analyzed sample and, ultimately, the reference population. Here a different interpretation is proposed, based on the fact that the indicators refer instead to a sample of events of participation in activities rather than to the individuals that those events produce. In other words, we try to show that the usual interpretations of these indicators are mostly incorrect.
\end{abstract}

Keywords: time use survey, indicators of participation, events, individuals

\section{Introduction}

The data obtained from time use surveys, starting with Gershuny's study (2000) on changes in the time use models adopted in post-industrial society, are used increasingly in social research. If we consider some recent articles, we can see the variety of topics covered, from the relationship between paid work, domestic work and gender (Blekesaune 2005; Dotti Sani 2012; Romano et al. 2012; Moreno-Colom 2015; Powell and Craig; 2015, Tézli and Gauthier 2009), to adolescents' time use (Zick 2010), to leisure time activities (Jarosz 2016; Stalker 2011), religious practices (Brenner 2011, 2012, Rossi and Scappini 2013, 2014), to name but a few.

An issue that deserves to be addressed and which usually tend to be neglected by literature concerns how time use data are interpreted. Indeed, the indications of some authors who have highlighted that the daily variability on how people use time raises questions about what the data on the use of time measure (Frazer and Stewart 2012; Kang and Darren 2010; Stewart, 2013), are still not taken into account. In addition to the interpersonal variability in the use of time, there is also the intrapersonal variability, so some activities can be not reported because they are not carried out on reference period of the data or vice versa, with the consequent tendency to underreport 
or overreport.

The aim of the article is not to delve into the questions that derive from the methodological point of view from intrapersonal variability, for which reference should be made to the few articles published on the subject. For example, Stewart (2013), which deals with the distortions generated by the procedures used in the analysis of data on the use of time because it may appear that zero time has been dedicated to activities, instead, carried out. Jara-Díaz and Rosales-Salas (2014) who deal with the most appropriate duration of time diaries.

The article is intended to be a sort of cautionary tale for users of time use data who consider them as data referring to individuals (emblematic is the use of regression or indicators usually used) while, instead, we maintain that they provide information on events (Rossi 2008), with consequences in terms of research and interpretation of data, as will be shown.

The aim is neither to study a concrete case (refer to studies on religious practice by Rossi and Scappini, 2013, 2014) to empirically support that data are interpreted in a way that it can be considered at least imprecise if not incorrect. The starting point is the question underlying the methodological articles of Frazer, Stewart (2012), Kang and Darren (2010) and Stewart (2013): what is actually measured with the data on time use? We aim to contribute to the development of a theoretical and conceptual framework supporting a different, and more precise, interpretation of time use data that considers what they effectively refer to, that is, as mentioned, events.

We are aware that we are moving in an unexplored territory (as demonstrated by the very limited, if not almost non-existent, the literature on the topic), but it is precisely the increasing use of data on time use that makes it necessary to open a reflection on what they measure and how they are analyzed.

\section{Types of diaries, main indicators and measuring participation}

The participation or otherwise in an activity is the starting point of any time use analysis. However, we have to ask what time use surveys actually measure when talking about participation.

One aspect to keep in mind when analyzing data on time use is that the data are collected from two samples of individuals who are independent. On the one hand, the sample of individuals who have carried out an activity or not and, on the other hand, the sample of those who have carried out an activity or not on the day on which they compile their diary. The two samples generate, as will be shown, what can be called a 'space of events', with all the consequences that this has on data analysis. For example, in the case of participation, does the indicator measure the one related to individuals or events? To address this issue, it is worth offering a brief overview of 
some methodological aspects of time use surveys, i.e., the tools used to collect information and the main indicators used.

Gathering information on time use poses several methodological issues (see Colleoni 2004; Gershuny 2000) concerning, firstly, the tool used (questionnaire or diary) to collect the data. Orientation leans clearly in favor of diaries, which have the advantage of allowing more precise information collection on the activities undertaken and the time dedicated to them compared to a questionnaire (Gershuny 2000). As they require more effort in being filled in, however, this leads to a high non-response rate.

The use of diaries involves choices concerning aspects such as the period of the diaries, the minimum period considered in the diaries (usually 5, 15 or 30 minutes), the classification of activities, the performance of more than one activity at the same time, the collection of information through interviews or self-compilation.

Without investigating these aspects any further, it is enough to consider some characteristics of diaries in order to hypothesize that the information collected concerns events and not individuals. They are:

- the duration of the diary;

- the days of the week on which the diary is compiled;

- the length of the period during which the data are collected, that is the time coverage. Duration of a diary refers to the length of the period considered, which may be one or more days, a week, a month, a year (Colleoni 2004; Gershuny 2000). Diaries with short duration (often only one day) are preferred as this increases the quality of the information, for easily understandable reasons. Indeed, in a daily diary are indicated the activities undertaken over 24 hours. If, on the other hand, the diary is weekly, it refers to the whole week, which may be less precise, as the memory may be fuzzier.

Another issue concerns the choice of the day on which the diary is filled in. In order to increase the precision and completeness of the information collected and reduce nonresponses, diaries are usually filled in referring to a sample day of the week. Even though in some cases the interviewees are asked to compile the diary on several days or every day of the week, the most common practice involves dividing the sample of interviewees into sub-samples, each of which compiles the diary only on a specific day. For example, in Italy Istat (the Italian National Institute of Statistics) splits the families in the sample into three groups, each of which is asked to compile the diary on different days (weekdays, Saturday, Sunday).

While in this way it is possible to collect information on the activities done on a given day, it, however, excludes the possibility of knowing how an individual organizes his activities over a week (Colleoni 2004). Indeed not all the activities are done every day. Moreover, the organization of time during the day may be part of given weekly planning. For example, an individual may not go shopping or do sports every day. 
Consequently, from the research conducted it has emerged that the differences between individuals in terms of activities done tend to be higher if the considered time span is shorter (for example if we refer to a day rather than a week) (Gershuny 2000).

Finally, time coverage refers to the period during which the diaries are collected. Possible options are every day in a year, six months or a shorter time span. An extended coverage, possibly annual, considers more the cyclical nature of some activities which are often linked to the seasons.

In time use data analysis are used principally two methods: regression and the use of participation and duration indicators. This article focuses on the latter.

As regards the first (see, for example, Díaz-Méndez and García-Espejo 2014; Flood, Moen 2015; Jarosz 2016; Powell and Craig 2015; Stalker 2011; Tézli and Gauthier 2001 ) it is sufficient to highlight that the use of regression requires a reference to individual data. Indeed, usually through regression, researchers try to understand how individual (social and personal, as well as other) variables affect participation in an activity and the time spent on that activity. It, therefore, assumes that there is a distinction between who does a given activity and who does not. However, the fact that the literature does not take into account the distinction between events and individuals means that the distinction between those who participate in an activity and those who do not is not straightforward. We will come back to this at the end of the next paragraph.

As regards the participation and duration indicators, we will concentrate on three of the most commonly used indicators in empirical research (see, for example, Charmes 2015; Istat 2016), which are:

- the generic average duration $(\mathrm{Ga})$ of an activity done, i.e., the ratio between the total time spent on the activity by the whole sample and the number of diaries compiled (total persons surveyed);

- the specific average duration $(S a)$ of an activity, i.e., the average time spent on the activity referring only to those who indicated they had done it in the diary;

- the frequency of participation, i.e., the percentage of persons who do a given activity. Since the purpose of this paragraph is to reflect on what time use surveys measure when it comes to participation, we will focus on the third indicator, the frequency of participation, leaving the discussion of the first two for the next paragraph. In our view, this is an ambiguous indicator. More precisely, as we will try to demonstrate, while it is usually interpreted as an indicator referring to individuals, it refers to events. This indicator can be of two types: relative to a single type of day (Monday, Tuesday, etc.), or an average day, generally working or weekday.

For example, Romano et al. (2012) state that there are strong differences in the contribution to domestic work by children in Italy according to gender which tend to 
increase with age. The frequency of participation in domestic work of children on an average day in 2002-2003 in the age bracket from 3 to 10 years is $28.3 \%$ for males and $35.4 \%$ for females, and increases with age up to respectively $43.1 \%$ and $78,9 \%$ in the age bracket 24 and over.

Another research on changes in meal consumption models in Spain and the United Kingdom (Díaz-Méndez and García-Espejo 2014) recorded that in Spain in 2010 a frequency of participation in the consumption of meals at home was $99 \%$, that of preparation and cooking of food was $66 \%$, and that of eating out was $25 \%$.

We may ask: when in the first example it is stated that $28.3 \%$ of males aged between 3 and 10 participate in domestic work, does it implicitly mean that $71.7 \%$ do not do this activity? In more general terms, we wonder what the exact meaning of 'frequency of participation is.'

An example helps to clarify the point. Let us suppose to have a sample of 1,000 respondents who fill in a daily diary for two days, and we study activity $\mathrm{x}$ (for example, doing sport) done on two days (Monday and Tuesday). We can hypothesize that in a given population $10 \%$ of individuals do the activity on both days, $30 \%$ only on Monday, $24 \%$ only on Tuesday and 36\% never do it.

These data allow us to calculate different indicators. We may state that the rate of participation in the activity — which here we will also call frequency rate - is $40 \%$ on Monday and $34 \%$ on Tuesday. Of course, we can also refer to these two values as presence rates, meaning that merely participating in activity also means being present at it. We can also calculate frequency rates relating to the two days together: if we consider those who are active in one or both days, we know that they constitute $64 \%$ of the population; if we consider exclusively those active on both days, we see that the value is $10 \%$.

Now let us suppose that we analyze the same population using a daily diary and a total sample of 2,000 interviewees (1,000 filling in the diary on Monday and 1,000 on Tuesday). Also, in this case, the frequency or presence rate for Monday is $40 \%$ $(400 / 1000)$ and for Tuesday 34\% (340/1000). As the interviewees fill in the diary either on Monday or on Tuesday, it is not possible to determine the frequency rate for at least one day or those active on both days. We can, however, calculate the presence rate on an average day, equal to $37 \%$ (740 diaries with a presence at activities out of the 2,000 total diaries). We can easily see that this rate is merely the average of the presence rates for the single days ( $40 \%$ and $34 \%$ respectively). This value coincides with the 'frequency of participation' which is usually calculated in surveys on time use.

Why do we record this significant deviation between the frequency rate for at least one day in the population ( $64 \%$, first example) and this average presence rate $(37 \%)$ ? 
Because not all those who are active are interviewed on the day they are active. So in calculating the average daily frequency, we apply weights which, in the presented example, are 1 for those who are active on both days and 0.5 for those who are active on only one day. Generally, in weekly diaries, the weights go from $1 / 7$ for those who are active only one day of the week to 1 for those who are active every day of the week. Similarly, for annual diaries, the weights go from 1/365 for those who are active only one day to 1 for those who are active every day.

Now we can answer the question posed earlier: what is the exact meaning of 'frequency of participation'? Knowing the distribution of the activity among the population, we can separate this rate into three parts: $10 \%$ who attend both days, $15 \%$ who attend only on Monday, $12 \%$ who attend only on Tuesday. Similarly, the "nonfrequency' rate $(63 \%)$ consists of 3 parts: $15 \%$ who attend on Monday but fill in the diary Tuesday, $12 \%$ who attend on Tuesday but fill in Monday and the remaining 36\% who effectively did not attend on either Monday or Tuesday. Therefore, the frequency of participation does not separate participants and non-participants.

The ambiguity of the indicator is not only a question of terminology (presence vs. frequency) but lies at a deeper level, concerning the confusion between statistics based on individuals and statistics based on events. Each interviewee fills in a diary, and in doing so communicates information concerning a specific activity: if he/she did it (positive event) or did not (null event). When we determine the frequency of participation in the hypothetical example, we consider 2000 events which constitute the 'space of events' for that activity. In that space, 740 events are positive, and 1,260 are null. The confusion originates from the fact that different individual produces every event. If we get confused, it is because we forget that the space of events is the sum of two independent samples of individuals, one relating to Monday and one relating to Tuesday, that generate a sample of diaries. If the survey had made recourse to only one sample of 1000 people who filled in the diary on both Monday and Tuesday, we could have been able to count how many of them had performed the activity on Monday and/ or on Tuesday in the 'space of individuals.' In one case the statistics are based on events, and in the other on individuals.

Therefore, the frequency of participation is an ambiguous indicator that refers to events and not to individuals. Moreover, it does not separate participants and non-participants, and this has significant consequences in terms of research. Indeed, the frequency of participation is used as dependent variable by who carry out a multivariate analysis to distinguish participants from non-participants.

For example, Brenner (2012) dealt with overreporting in religious practice in Canada. He defined it as the difference between the values of participation in mass obtained through diaries on time use (the frequency of participation) and the traditional question 
asked in surveys ("Other than on special occasions -such as weddings, funerals or baptisms-, how often did you expect religious services or meetings in the last 12 months?"). To explain overreporting, he referred to the value individuals give to religious identity, which can be a source of distortion when they respond to a survey. In our opinion, rather than the sociological reasons identified by Brenner, overreporting is due to methodological reasons. More precisely not so much to a measurement error, but to the comparison between indicators that are related to events (data on time use) and individuals (survey) (for a more in-depth analysis on the subject, see Rossi and Scappini 2013, 2014). Indeed, the boundaries between those attending mass and those who do not, are not clear. For example, within those who regularly attend mass, not everyone fills in the diary on the day they went to mass. In this way, however, they end up being among the non-participants. Thus some activities are not reported just because not carried out when the diary is filled in.

\section{Indicators of participation and duration}

The distinction between data referring to events and individuals implies that, in contrast to what is usually done in time use data analysis, it is possible to distinguish between indicators relating to the first and the second. Now we will address this diversity of indicators systematically. For this purpose, we may consider the example presented in table 1 that shows the differences between the values of the indicators of events and individuals can be more or less accentuated.

Let us suppose we used a diary with weekly coverage and duration, so the data in the table represent five hypothetical results relating to six individuals $(q 1, q 2, q 3, q 4, q 5$, q6) who fill in a diary for each day of the week relating to a generic activity. Each cell presents the duration of an event; if this is greater than zero, it corresponds to the existence of a positive event. If the value is zero (null event), on the other hand, clearly the interviewee has not done the considered activity. For each hypothesis, the table contains 42 pieces of information relating to events (and more precisely the duration of an event) referred to the six interviewees, which contribute to determining that which may be defined the space of the events (a $6 \times 7$ matrix, 6 respondents for 7 days of the week).

The data in table 1 can, in any case, be seen as the results of a survey of diaries with weekly coverage but daily duration. In this second case, we refer to 7 independent samples, one for each day, each one formed of six subjects. Of course, the data in a particular row ( $q 1$ or $q 2$, etc.) no longer refer, as happened in the previous situation, to the same individual, but instead represents the response of each first $(q 1)$, second $(q 2)$, etc. subject in each sample (clearly, the last two columns in table 1 no longer have any meaning). 
For each hypothesis in the table, we can calculate different indicators for participation, presence, and average duration both for individual days and for average days.:

- $\mathrm{t}$, the rate of participation (or frequency) in the period: the people active on at least one day. It refers only to individuals. The numerator is calculated by counting the number of rows containing at least one event. In hypothesis $b$ the rate is equal to $83.3 \%(5 / 6)$;

- $\mathrm{t} 2$, the rate of daily presence/frequency: the rate between active people and the total number of interviewees every day. It refers both to interviewees and events, given the one-to-one correspondence between the two. In hypothesis $b$ Monday is equal to $50 \%$ $(3 / 6)$;

- $\mathrm{t} 3$, the rate of average presence or density of the events: the ratio between the recorded events (those indicated with a value other than zero) and the total number of theoretically possible events. Again in hypothesis $b$, it is equal to $35.7 \%$ (15/42). The indicator refers only to events.

The main indicators of duration are:

- d1, specific average duration dedicated to the activity by active individuals: this refers to individuals active on at least one day of the week. In hypothesis $b$ it is equal to 8.6 minutes;

$-\mathrm{d} 2$, specific average duration of events: is the ratio between the total duration of the events in a week and the number of events with a duration other than zero. In hypothesis $b$ it is equal to 20 minutes $(300 / 15)$;

- $d 3$, generic average duration: is the ratio between the total duration and the number of theoretically possible events. In hypothesis $b$ this is equal to 7.1 minutes $(300 / 42$ referring to events, 42.9/6 referring to individuals);

- d4, specific average duration by type of day: this is the ratio between the total duration of an activity in a day and the number of individuals active or events observed. In hypothesis $b$ and for Monday this is equal to 26.7 minutes (80/3); it refers to both individuals and events (see t2);

- d5, generic average duration by type of day: this is the ratio between the total duration of an activity in a day and the total number of individuals interviewed (active or inactive) or possible events. In hypothesis $b$ and for Monday this is equal to 13.3 minutes (80/6); it refers to both individuals and events. 
Table 1. Five hypothetical results of measurements with six weekly diaries

Mon Tues Wed Thu Fri Sat Sun Tot (N) Average Number

Events duration of

activity

days

\begin{tabular}{llllllllllll}
\hline$H$ & $\mathrm{~d} 1$ & 10 & 10 & 10 & 10 & 10 & 10 & 0 & 60 & 8.6 & 6 \\
$y$ & $\mathrm{~d} 2$ & 10 & 10 & 10 & 10 & 10 & 10 & 0 & 60 & 8.6 & 6 \\
$p$ & $\mathrm{~d} 3$ & 10 & 10 & 10 & 10 & 10 & 10 & 0 & 60 & 8.6 & 6 \\
$t$ & $\mathrm{~d} 4$ & 10 & 10 & 10 & 10 & 10 & 10 & 0 & 60 & 8.6 & 6 \\
$h$ & $\mathrm{~d} 5$ & 10 & 10 & 10 & 10 & 10 & 10 & 0 & 60 & 8.6 & 6 \\
$s$ & $\mathrm{~d} 6$ & 0 & 0 & 0 & 0 & 0 & 0 & 0 & 0 & 0 & 0 \\
$s$ & & & & & & & & & & &
\end{tabular}

$a$

\begin{tabular}{|c|c|c|c|c|c|c|c|c|c|}
\hline Tot. 50 & 50 & 50 & 50 & 50 & 50 & 0 & 300 & $(30$ & 5.0 \\
\hline$H \quad d 1 \quad 60$ & 0 & 0 & 0 & 0 & 0 & 0 & 60 & & \\
\hline
\end{tabular}

$\begin{array}{llllllllllll}y & \mathrm{~d} 2 & 0 & 60 & 0 & 0 & 0 & 0 & 0 & 60 & 8.6 & 1\end{array}$

$\begin{array}{llllllllllll}o & \mathrm{~d} 3 & 0 & 0 & 60 & 0 & 0 & 0 & 0 & 60 & 8.6 & 1\end{array}$

$\begin{array}{llllllllllll}t & \mathrm{~d} 4 & 10 & 10 & 10 & 10 & 10 & 10 & 0 & 60 & 8.6 & 6\end{array}$

$\begin{array}{llllllllllll}e & \mathrm{~d} 5 & 10 & 10 & 10 & 10 & 10 & 10 & 0 & 60 & 8.6 & 6\end{array}$

$\begin{array}{llllllllllll}i & \mathrm{~d} 6 & 0 & 0 & 0 & 0 & 0 & 0 & 0 & 0 & 0 & 0\end{array}$

$s$

$b$

\begin{tabular}{llllllllllll} 
Tot. & 80 & 80 & 80 & 20 & 20 & 20 & 0 & 300 & $(15)$ & 42.9 & 2.5 \\
\hline
\end{tabular} 


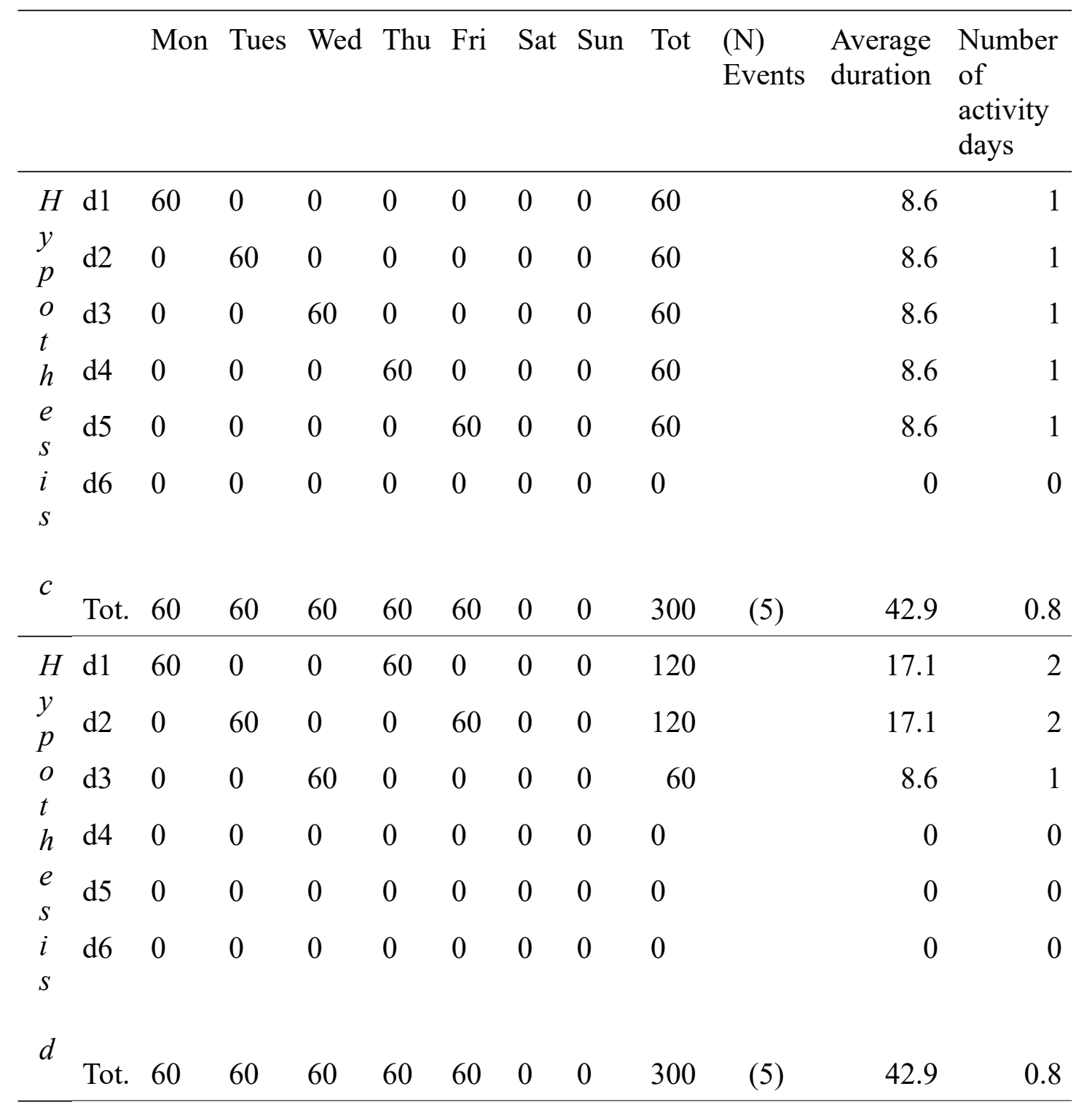




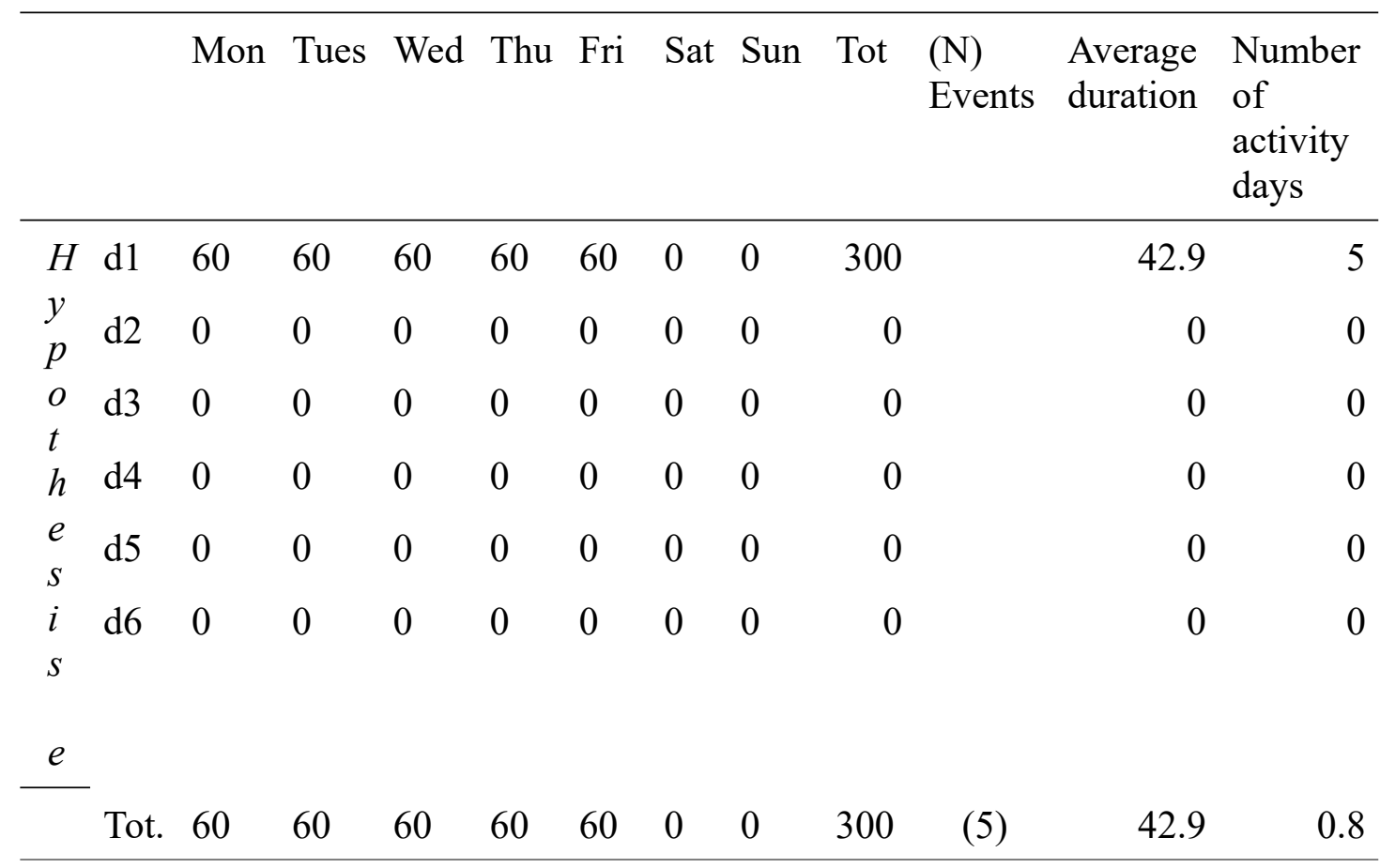

Table 2 shows the results relating to all indicators identified for each of the hypotheses contained in table 1.

As can be seen in table 2, the values of the indicators relating to individuals differ, at times significantly, from those based on events. Thus the participation rate (or frequency) ( $\mathrm{t} 1$ ) of the samples considered in the hypotheses $a, b$ and $c$ is $83.3 \%$ in all three cases, while the density of the events ( $\mathrm{t} 3$, also presence on an average day) not only has lower values but also varies consistently from one hypothesis to another: $71.4 \%$ in $a, 35.7 \%$ in $b, 11.9 \%$ in $c$. Again, the specific duration on an average day (d1) in the hypotheses $a, b$ and $c$ is 8.6 minutes while the duration of the events on an average day (d2) varies significantly from one hypothesis to another: 10 in $a, 20$ in $b$ and 60 in $c$. In each of the three hypotheses considered here, the total amount of time devoted to the activity is 300 minutes, the active subjects for at least one day are 5 , each subject is active for 60 minutes per week. 
Table 2. Indices of average duration (minutes) and participation (\%) in activity $X$ in five hypothetical survey results using weekly diaries; $(N)=6$

Average day weekly

Mon Tues Wed Thur Fri Sat Sun Events Individuals

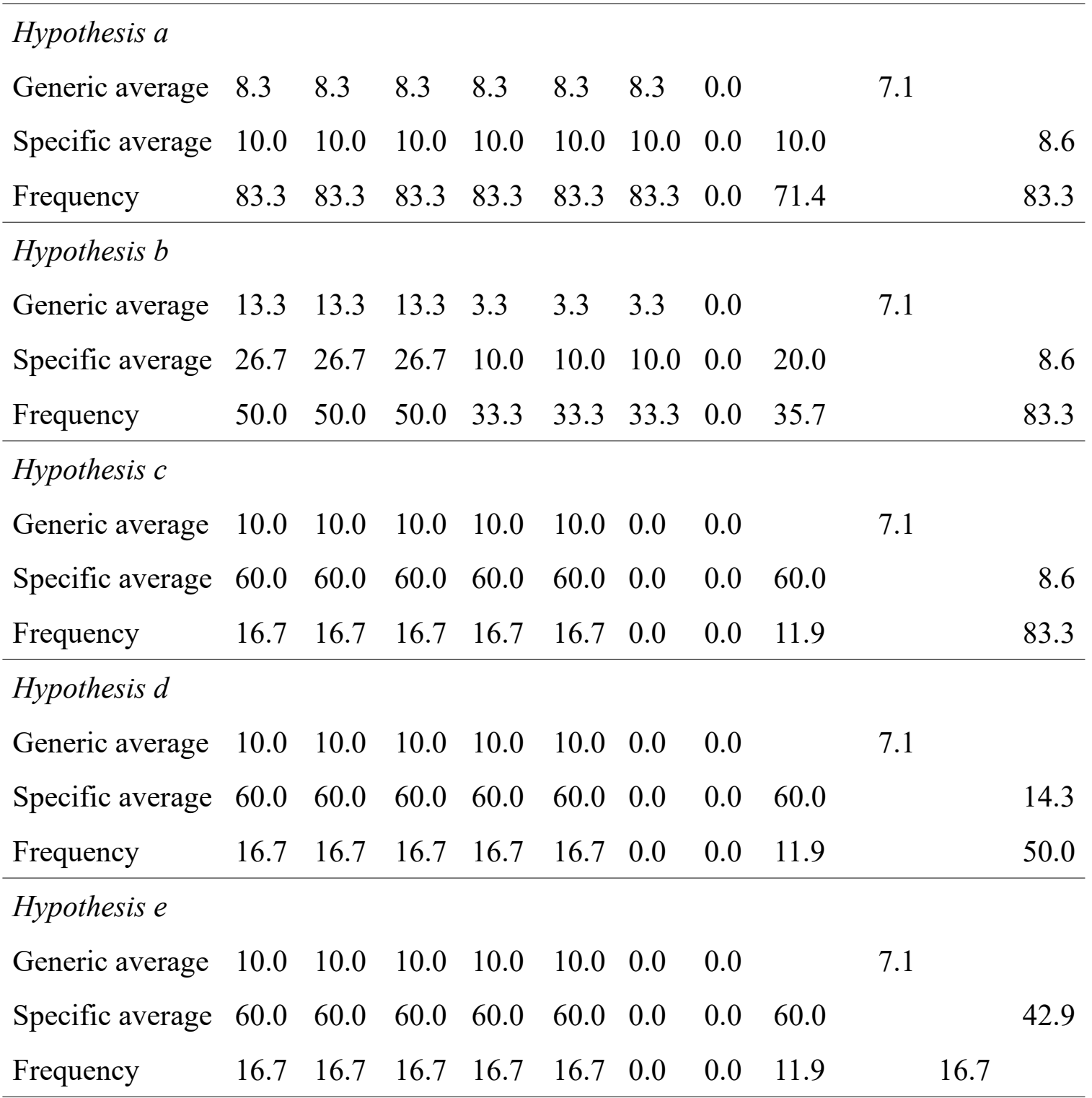

Source: own elaboration from Istat (2007) 
The more or less accentuated deviation between the values of the events indicators and the individuals' indicators clearly depends on the degree of concentration or dispersion of the weekly times of the activities performed by the subjects on different days of the week. Indeed, in hypothesis $a$ the five active subjects spend a total of 60 minutes for the activity, distributing it equally over the six days (10 minutes a day); in hypothesis $b$ three of them prefer to concentrate the 60 minutes total in one single day, while in hypothesis $c$ the concentration in a single day concerns all active subjects. The concentration of the activity thus has the effect of reducing the number of positive episodes in the space of events, thus reducing that which in table 2 we indicated as the 'frequency' of events ( $\mathrm{t} 3: 71.4 \%, 35.7 \%$, and $11.95 \%$ respectively in hypotheses $a, b$ and $c$ ) and increasing the specific duration of the average day (d2: 10, 20, 60 minutes respectively in $\mathrm{a}, \mathrm{b}, \mathrm{c})$.

For a clear explanation, thus far we have examined an essentially theoretical position: that of a survey with weekly duration and weekly coverage, relating to a single week of the year.

At the beginning of the paragraph, however, we mentioned that table 1 could be seen as the representation in the form of the space of events of a survey with daily duration and weekly coverage, the most common situation in empirical surveys. In this case clearly, it is not possible to calculate the values contained in the column 'average durations' and 'active/not active': they serve no purpose, as each row contains data relating to 7 different subjects. Consequently, the indicators based on individuals in table 2 cannot be calculated.

Therefore the available indicators belong to what we called event statistics, with the sole exception of the 'generic average duration' ( $G a$, the ratio between total activity time and the total number of positive and null events). We have already seen in tab. 2 , its value does not change when calculated as a statistic of individuals, rather than of events.

On the other hand, the "participation frequency" ( $F$, ratio between positive events and total possible events) is, as we have also demonstrated in the previous paragraph, an ambiguous indicator that is not easy to interpret: its value is influenced both by the number of subjects performing the activity but also the way in which they perform it. The more the activity of the individuals is concentrated in a few days, the lower the value, and vice versa. Table 2 showed that the variations of this measure are very high, even in the presence of populations with identical total duration and an equal number of active subjects. It is certain that in no way it can be used to estimate the participation rate of a population. For this reason, we have suggested here that we refer to the indicator as the density of events, a terminology that accurately expresses its 
content and avoids confusion with a participation rate.

The crucial third indicator, the 'specific average duration' ( $\mathrm{Sa}$, the ratio between total time devoted to an activity and the number of positive events), presents the same ambiguities as the previous one. We can consider that the three indicators (with equal days, single, weekday, average) are linked to the ratio: $G a=S a * F$. With equal total time devoted and the number of active subjects, $G a$ is constant. On the other hand, $F$ is not: as we have seen, its value is inversely proportional to the concentration of the activities into individual days (of the week).

As we have tried to show, therefore, the indicators of the events and those of the individuals must be distinguished. Which is not usually done in the literature. Not only, but indicators calculated on events are considered as indicators referred to individuals. For example, in a survey to measure the time spent by individuals on sports and exercises activities in the United States (Woods 2017), the specific average is used. In the methodological note (Woods 2017: 27) it is written that: "The average number of hours per day for persons who did the activity is computed using only the responses from those who engaged in a particular activity on their diary day." Then comparisons are made based on variables such as gender and age group which, since the data refer to events and given the ambiguity that the indicator presents, do not make sense in our opinion.

\section{Time cycles of repetition}

A final aspect to be considered about the indicators used in time use data analyses concerns the time cycle of some activities. By this, we mean that various activities are repeated over time according to cycles which may be different. Some activities are repeated daily, such as eating and sleeping. Others may have a longer cycle, such as every two or three days in the week (for example, doing sport) or once a week (for example, going to the cinema) or a month (for example, going back to your home town if you study away from home). Finally, some activities are affected by seasonality (for example, going to school).

These aspects must be considered when interpreting the indicators.

For example, let us imagine activities carried out with different repetition cycles but by almost all the population. In the case of activities with a daily repetition cycle, the rate of presence or density (or frequency of participation, to use the most common name) has little sense as it will tend to be equal to $100 \%$. The only indicator affected is the generic average duration which coincides with the specific average duration $(G a=S a * F$ and in this case $F=1)$.

In the case of activities undertaken less regularly and therefore with a repetition time cycle other than one day, on the other hand, the rate of density will be less than $100 \%$ 
as the activity is not done every day. Moreover, in the case of diaries compiled daily, even some people who do the activity may be interviewed on a day on which they are inactive, and this lowers the average frequency to below its actual value. The deviation between the two values is as great as the length of the time cycle of repetition of an activity. It is clear that if an activity is done on a monthly time cycle, there is a higher probability of interviewing people who do a given activity on the day on which they are not active compared to an activity with a shorter repetition cycle, for example, every other day.

To illustrate the effects of time discontinuity on the indicators that usually used, we consider the data on an activity about which we know, even if not exhaustively, some important seasonal and non-seasonal variations: we refer to school attendance.

School attendance (in Italy) is characterized by a seasonal time cycle (frequency from September to June) and by two daily cycles (in some schools frequency is from Monday to Saturday, in others from Monday to Friday). In order to make the contrast of meanings of the frequency of participation indicator more concretely clear, we will, therefore, examine the data relating to school attendance in compulsory schooling. Table 3 shows the three traditional statistics (with the common Istat names) referring to the events for the 7-13 year age group. These data offer two advantages. First of all, there is a sure frequency data with which to compare the results of the daily diaries. In compulsory school, everyone has to attend, so the frequency is $100 \%$. Secondly, because this, like many other activities (probably the majority), is characterized by a complex time cycle.

Observing table 3 we can make statements such as: "on an average weekday compulsory school frequency involves $58.4 \%$ of the 7 to 13 age population". Alternatively: "on an average weekday the percentage of participation increases to $71.2 \% "$. There is indeed something wrong here: at least on weekdays, the average rate of attendance should be $100 \%$, not $71.2 \%$.

The objection that it refers to an average rate of frequency which, therefore has a value close to $100 \%$ on school days and $0 \%$ on holidays, does not seem convincing.

The difference between the frequency rate, which should be close to $100 \%$, and the average rate $(71.2 \%)$ is too high not to raise questions. It induces us to assume that $28.8 \%$ of interviewees compiled the diary on holiday or a day they were not at school.

It may lead to problems of interpretation if the data are considered as referring to individuals. For example, we may imagine that there are distortions in the sub-samples of interviewees linked to the time cycles of the activities. The differences according to gender presented in table 3 are emblematic. It is hard to explain the fact that the frequency of males is higher than females from Monday to Wednesday and Saturday and the frequency of females is higher on Thursday and Friday. 
Table 3. Istat indices of average duration (minutes) and participation (\%) concerning school attendance by day of the week and compilation of the diary and gender; population aged 7 to 13. Istat Time use 2002-03

Mon Tues Wed Thur Fri Weekday Sat Sun Tot.
average average

\begin{tabular}{|c|c|c|c|c|c|c|c|c|c|}
\hline Generic average & 229 & 233 & 259 & 255 & 254 & 240 & 146 & 0 & 193 \\
\hline Specific average & 329 & 334 & 348 & 330 & 343 & 337 & 290 & 40 & 331 \\
\hline Frequency & 69.4 & 69.8 & 74.6 & 77.3 & 65.4 & 71.2 & 50.4 & 0.2 & 58.4 \\
\hline$(\mathrm{N})$ & 647 & 556 & 547 & 537 & 540 & 2,827 & 566 & 541 & 3,934 \\
\hline \multicolumn{10}{|l|}{ Males } \\
\hline Generic average & 239 & 249 & 268 & 241 & 216 & 242 & 149 & 0 & 196 \\
\hline Specific average & 336 & 345 & 345 & 324 & 346 & 339 & 290 & 0 & 333 \\
\hline Frequency & 71.0 & 72.3 & 77.5 & 74.3 & 62.5 & 71.4 & 51.5 & 0.0 & 58.9 \\
\hline (N) & 334 & 256 & 311 & 268 & 301 & 1,470 & 293 & 275 & 2,038 \\
\hline \multicolumn{10}{|l|}{ Females } \\
\hline Generic average & 218 & 219 & 248 & 270 & 233 & 236 & 143 & 0 & 190 \\
\hline Specific average & 322 & 324 & 351 & 336 & 338 & 333 & 291 & 40 & 328 \\
\hline Frequency & 67.7 & 67.4 & 70.8 & 80.6 & 68.8 & 70.9 & 49.1 & 0.4 & 57.9 \\
\hline$(\mathrm{N})$ & 313 & 301 & 236 & 268 & 240 & 1,358 & 273 & 266 & 1,897 \\
\hline
\end{tabular}

Source: own elaboration from Istat (2007)

On the other hand, we might think that the time cycle of repetition of activities affects the selection of persons interviewed and we may hypothesize that the sociodemographic characteristics of the subjects in the two sub-samples (interviewed on the day of frequency and interviewed on holidays or days of absence) are not necessarily the same. A certain amount of caution would be needed in interpreting data as if 
referred to individuals.

\section{Conclusions}

The statement that data on time use do not refer to individuals but to events is based on considerations that begin with how the information is collected. As we have seen in paragraph 1, the concept of participation in an activity is used in a somewhat ambiguous manner. In the calculation of the frequency indicators of participation in an activity, there is no clear distinction between those who have carried out an activity and those who have not. Being inactive may mean never doing an activity or not doing an activity on the day the diary is compiled. It is clear that these are very different situations and only in the first situation is there a lack of participation.

In paragraph 2, examples have been used to illustrate the difference between indicators of events and individuals. The distinction between the two types of indicators allows a more precise interpretation of the time use, since equal values in the indicators relating to individuals may correspond to different values in those relating to events and vice versa.

Finally, as we have seen in paragraph 3, the reliability of the calculated values of frequency of participation is at least doubtful in the presence of cycles and temporal discontinuities of the activities examined, even more so when these values are used to compare sub-samples (such as males and females, young people and the elderly, etc.).

\section{References}

Blekesaune, Morten. 2005. Working Conditions and Time Use. Acta Sociologica 48(4): 308-320.

Brenner, Philip S. 2011. Identity Importance and the Overreporting of Religious Service Attendance: Multiple Imputation of Religious Attendance Using the American Time Use Study and the General Social Survey. Journal for the Scientific Study of Religion 50(1):103-15.

Brenner, Philip S. 2012. Identity as a Determinant of the Overreporting of Church Attendance in Canada. Journal for the Scientific Study of Religion 51(2):377-385.

Castegnaro, Alessandro and Dalla Zuanna, Gianpiero. 2006. Studiare la pratica religiosa: differenze tra rilevazione diretta e dichiarazione degli intervistati sulla frequenza alla messa. Polis 1/2006:85-112. 
Charmes, Jacques. 2015. Time Use Across the World: Findings of a World Compilation of Time Use Surveys. New York: UNDP Human Development Report Office.

Colleoni, Matteo. 2004. I tempi sociali. Teorie e strumenti analisi. Roma: Carocci.

Díaz-Méndez, Cecilia and García-Espejo, Isabel. 2014. Eating Practice Models in Spain and the United Kingdom: A Comparative Time-Use Analysis. International Journal of Comparative Sociology 55(1):24-44.

Dotti Sani, Giulia Maria. 2012. La divisione del lavoro domestico e delle attività di cura nelle coppie italiane: un'analisi empirica. Stato e Mercato (1):161-194.

Flood, Sarah M. and Moen, Phyllis. 2015. Healthy Time Use in the Encore Years: Do Work, Resources, Relations, and Gender Matter? Journal of Health and Social Behavior 56(1):74-97.

Frazis, Harley and Stewart, Jay. 2012. How to Think about Time-Use Data: What Inferences Can We Make about Long- and Short-Run Time Use from Time Diaries?, Annals of Economics and Statistics Issue 105-106:231-245.

Gershuny, Jonathan. 2000 Changing times. Work and Leisure in Postindustrial Society. Oxford, New York: Oxford University Press.

Istat. 2007. Uso del Tempo Anno 2002-2003 [computer file]. Rome, Italy: Istituto Nazionale di Statistica [producer and distributor].

Istat. 2016. I tempi della vita quotidiana. Statistiche report 1-26.

Kang, Hejun and Scott, Darren M. 2010. Exploring day-to-day variability in time use for household members. Transportation Research Part A, 44:609-619

Jara-Díaz, Sergio and Rosales-Salas, Jorge. 2015. Understanding time use: Daily or weekly data? Transportation Research Part A, 76:38-57.

Jarosz, Ewa. 2016. The Duration and Dynamics of Leisure among the Working Population in Poland. A Time-Use Approach. World Leisure Journal 58(1):44-59.

Moreno-Colom, Sara. 2015. The Gendered Division of Housework Time: Analysis of Time Use by Type and Daily Frequency of Household Tasks. Time \& Society 0(0):125.

Powell, Abigail and Craig, Lyn. 2015. Gender Differences in Working at Home and Time Use Patterns: Evidence from Australia. Work, Employment \& Society 29(4):571589. 
Romano, Maria Clelia, Mencarini, Letizia and Tanturri, Maria Letizia. A cura di. 2012 Uso del tempo e ruoli di genere. Tra lavoro e famiglia nel ciclo di vita. Roma: Istat.

Rossi, Maurizio. 2008. Leggere i dati Istat sull'uso del tempo. Polis (2):275-303.

Rossi, Maurizio and Scappini, Ettore. 2013 Possiamo ancora dirci cristiani? La volatilità della partecipazione individuale alla messa. Polis 27(3):363-394.

Rossi, Maurizio and Scappini, Ettore. 2014. Church Attendance, Problems of Measurement, and Interpreting Indicators: A Study of Religious Practice in the United States, 1975-2010. Journal for the Scientific Study of Religion 53(2): 249-267.

Stalker, Glenn J. 2011. A Widening Parental Leisure Gap: The Family as a Site for Late Modern Differentiation and Convergence in Leisure Time within Canada, the United Kingdom and the United States. Canadian Journal of Sociology/Cahiers canadiens de Sociologie 36(1):25-58.

Tézli, Annette and Gauthier, Anne H. 2009. Balancing Work and Family in Canada: An Empirical Examination of Conceptualizations and Measurements. Canadian Journal of Sociology/Cahiers canadiens de Sociologie 34(2):433-462.

Stewart, Jay. 2013. Tobit or Not Tobit?. Journal of Economic and Social Measurement 38(3):263-290.

Woods, Rose A. 2017. Sports And Exercise. U.S. Bureau Of Labor Statistics. Spotlight on Statistics 1-27.

Zick, Cathleen D. 2010. Adolescent Time Use. Youth \&Society 41(4):569-596.

\section{Corresponding author}

Marco Trentini, Department of Education Studies, University of Bologna, Via Filippo Re 6, 40126 Bologna, Italy. E-mail: marco.trentini@,unibo.it 